\title{
On Optimal Servosystem with Quadratic Stability
}

\author{
Hisashi TsuchIdA* and Nobuhide SudA**
}

\begin{abstract}
We discuss the construction of optimal servosystems with quadratic stability for uncertain systems with structured, time-invariant and bounded uncertainties. The aim is to design a control law such that the feedback system has three features : its output asymptotically tracks any constant reference input, it minimizes a certain quadratic performance index, thus inheriting the desirable properties of optimal regulator design, and the stability and the tracking property are both ensured for any uncertainty in a given bounded set. Based upon the observation that the quadratically stabilizing controller by Petersen, if exists, is an optimal regulator, in some way or another, for any of the uncertain system under consideration, such a control law together with a sufficient condition of its existence is derived. Further, by extending a particular optimal servosystem proposed by Suda and Ikeda to the uncertain system, it is shown that, under a sufficient condition, weaker than the so-called 'matching condition', it is possible to construct an optimal servosystem with quadratic stability which has a set of gain tuning parameters. Through an example, it is demonstrated that the speed of response is easily selected by adjusting these tuning parameters.
\end{abstract}

Key Words: optimal servosystem, quadratic stability

\section{Introduction}

In this paper, we consider the construction of optimal servosystems for uncertain systems with time-invariant and bounded uncertainties. The optimal servosystem under consideration is designed by the optimal regulator theory ${ }^{1)}$, or the linear-quadratic (LQ) optimal control, in such a way that its output asymptotically tracks a constant

* Mizushima Works, Kawasaki Steel Corporation, 1 Kawasakidori, Mizushima, Kurashiki

** Faculty of Engineering Science, Osaka University, 1-1 Machikaneyama, Toyonaka

(Received October 15, 1990)

(Revised March 20, 1991) reference input.

For uncertain systems a notion of 'quadratic stability' is introduced. Petersen ${ }^{2)}$ has derived, for quadratically stabilizable uncertain systems, an algorithm to construct a stabilizing controller based on a solution of a certain algebraic Riccati equation. We clarify the relationship between the LQ optimal controller and the controller obtained by this stabilization algorithm. It is readily shown, in Section 2, that the quadratically stabilizing controller is LQ optimal, in some way or another, for any of the uncertain system under consideration. This is the main motivation that we consider the optimal servosystem with quadratic stability.

In Section 3, we will obtain the optimal servosystem with quadratic stability, by directly applying Petersen's method ${ }^{2)}$ to the augmented system. Heretofore the robustness of optimal servosystems was considered only in a neighborhood of its nominal plant. As against this the optimal servosystem constructed by using the quadratically stabilizing controller can ensure the stability and the tracking property for any uncertainty in a given bounded set.

In Section 4, by extending a particular optimal servosystem shown in Ref. 3) to the uncertain system, we will show that, under a condition stated in Lemma 3 , an optimal servosystem which has a gain tuning parameter as well as quadratic stability can be constructed. Furthermore it will be shown that this condition is a sufficient condition for quadratic stabilizability weaker than the so-called 'matching condition'.

Here we define some notation used in this paper. $\mathbf{R}, \mathbf{R}^{n}$, and $\mathbf{R}^{n \times m}$ denote the set of all real numbers, all $n$-dimensional real vectors, and all $n \times m$ real matrices, respectively. For a matrix $A,\|A\|$ denotes the largest singular value, and for a stable 
real rational matrix $G,\|G(s)\|_{\infty}$ is defined as follows,

$$
\|G(s)\|_{\infty}:=\sup _{\omega \in \mathbf{R}}\|G(j \omega)\| .
$$

$\mathbf{F}^{p^{\times q}}$ is defined as

$$
\mathbf{F}^{p \times q}:=\left\{F \in \mathbf{R}^{p \times q} \mid\|F\| \leq 1\right\} .
$$

If a real symmetric matrix $R$ is positive (non-negative) definite then $R$ is denoted as

$$
R>0 \quad(\geq 0) \text {. }
$$

For two real symmetric matrices $P$ and $Q$,

$$
P>Q \quad(\geq Q)
$$

means $P-Q$ is positive (non-negative) definite.

\section{Definitions and Preliminary Results}

Before considering servosystems, we introduce the concept of quadratic stability for an uncertain system described as follows

$$
\Sigma_{0}: \dot{x}=(A+D F E) x+B u,
$$

where $x \in \mathbf{R}^{n}$ is the state and $u \in \mathbf{R}^{m}$ the control input, respectively, $A \in \mathbf{R}^{n \times n}, B \in \mathbf{R}^{n \times m}, D \in \mathbf{R}^{n \times p}$ and $E \in \mathbf{R}^{q^{\times n}}$ are given matrices, and $F \in \mathbf{F}^{p \times q}$ is a matrix of uncertain parameters, which is normalized by $D$ and $E$.

'Quadratic stabilizability' and 'quadratic stability' are defined as follows ${ }^{4}$.

【Definition 1】A system, $\Sigma_{0}$, is said to be 'quadratically stabilizable' via linear control if there exist an $m \times n$ real constant matrix $K$, an $n \times n$ positive definite symmetric matrix $P$ and a constant $\alpha>0$ such that for any $F \in \mathbf{F}^{p \times q}$, the closedloop system with state feedback $u=-K x$ and Lyapunov function $V(x)=x^{T} P x$ have the following property

$$
\begin{aligned}
L(x, t) & :=\frac{d V}{d t} \\
& =2 x^{T} P(A+D F E) x-2 x^{T} P B K x \\
& \leq-\alpha\|x\|^{2}
\end{aligned}
$$

for all pairs $(x, t) \in \mathbf{R}^{n} \times \mathbf{R}$.

【Definition 2】 Consider the autonomous system obtained by setting $u=0$. This autonomous system is said to be 'quadratically stable' if there exist an $n \times n$ positive definite symmetric matrix $P$ and a constant $\alpha>0$ such that for any $F \in \mathbf{F}^{p \times q}$, the system and Lyapunov function $V=x^{T} P x$ have the following property

$$
\begin{aligned}
L(x, t): & =\frac{d V}{d t} \\
& =2 x^{T} P(A+D F E) x \leq-\alpha\|x\|^{2} .
\end{aligned}
$$

The following two results give necessary and sufficient conditions for quadratic stabilizability and quadratic stability.

《Lemma 1》(Petersen ${ }^{2}$, Th. 3.1, 3.2, 3.3) Let $Q \in \mathbf{R}^{n \times n}$ and $R \in \mathbf{R}^{m \times m}$ be given positive definite symmetric matrices. Then $\Sigma_{0}$ is quadratically stabilizable if and only if there exists a constant $\varepsilon>0$ such that the Riccati equation

$$
\begin{aligned}
& A^{T} P+P A-\frac{1}{\varepsilon} P B R^{-1} B^{T} P+P D D^{T} P \\
& \quad+E^{T} E+\varepsilon Q=0
\end{aligned}
$$

has a positive definite symmetric solution $P$. If such a solution exists then a quadratically stabilizing control law is given by

$$
u=-\frac{1}{\varepsilon} R^{-1} B^{T} P x .
$$

Further the existence of such a solution to Eq. (4) does not depend upon the choice of $Q$ and $R$ and if there exists a constant $\varepsilon_{*}>0$ such that the Riccati equation (4) has a positive definite solution, then for any $\varepsilon \in\left(0, \varepsilon_{*}\right]$, the Riccati equation (4) has a positive definite symmetric solution $P$.

《Lemma 2》(Khargoneker, et al.5), Th. 2.7) Consider the autonomous system obtained by setting $u=0$. Then the system $\Sigma_{0}$ is quadratically stable if and only if it satisfies the following conditions

1) $A$ is a stability matrix.

2) $\left\|E(s I-A)^{-1} D\right\|_{\infty}<1$.

It is clear that the quadratically stabilizing control ( 5 ) is an LQ optimal control for the nominal system $(F=0)$. Is the control optimal for the uncertain system $\Sigma_{0}$, that is, $F$ is not necessarily zero but in $\mathbf{F}^{p \times q}$ ?

From Eq. (4), we get

$$
\begin{gathered}
P(A+D F E)+(A+D F E)^{T} P \\
-\frac{1}{\varepsilon} P B R^{-1} B^{T} P+Q_{P}(F)=0,
\end{gathered}
$$

where

$$
\begin{aligned}
Q_{P}(F)= & P D D^{T} P+E^{T} E-P D F E \\
& -(D F E)^{T} P+\varepsilon Q \\
= & \left(P D-E^{T} F^{T}\right)\left(P D-E^{T} F^{T}\right)^{T} \\
& +E^{T}\left(I-F^{T} F\right) E+\varepsilon Q .
\end{aligned}
$$

If $F \in \mathbf{F}^{p \times q}$, we have 


$$
Q_{P}(F)>0 \quad \forall F \in \mathbf{F}^{p^{\times q}} .
$$

From above, we have following fact.

[Fact 1] The quadratically stabilizing control (5) is LQ optimal for any $F \in \mathbf{F}^{p \times q}$ and the associated quadratic cost function is

$$
J=\int_{0}^{\infty}\left\{x^{T} Q_{P}(F) x+\varepsilon u^{T} R u\right\} d t .
$$

\section{Construction of Optimal Servosystems with Quadratic Stability}

Consider an $m$-inputs $m$-outputs uncertain linear system

$$
\Sigma: \dot{x}=(A+D F E) x+B u, \quad y=C x,
$$

where $x, u, A, B, D, E$ and $F$ are defined in Section 2, $y \in \mathbf{R}^{m}$ is the output and $C \in \mathbf{R}^{m \times n}$ a given matrix. Here we will construct an optimal servosystem with quadratic stability.

The servosystem, we consider here, has the structure shown in Fig. 1.

In Fig. 1, the control error signal is defined as

$$
e=r-y,
$$

and its integral as

$$
w=\int_{-\infty}^{t} e d \tau
$$

The state equation for the augmented system, the state of which is $\left[x^{T}, w^{T}\right]^{T}$, is obtained as follows

$$
\begin{aligned}
& \Sigma_{a}:\left[\begin{array}{c}
\dot{x} \\
\dot{w}
\end{array}\right]=\left[\begin{array}{cc}
A+D F E & 0 \\
-C & 0
\end{array}\right]\left[\begin{array}{l}
x \\
w
\end{array}\right]+\left[\begin{array}{l}
B \\
0
\end{array}\right] u+\left[\begin{array}{l}
0 \\
I
\end{array}\right] r \\
& y=\left[\begin{array}{ll}
C & 0
\end{array}\right]\left[\begin{array}{c}
x \\
w
\end{array}\right] .
\end{aligned}
$$

Define the deviation

$$
\begin{aligned}
& \delta x:=x-x_{\infty}, \quad \delta u:=u-u_{\infty} \\
& \delta w:=w-w_{\infty}, \quad \delta y:=y-y_{\infty} \\
& \delta \tilde{x}:=\left[\begin{array}{c}
\delta x \\
\delta w
\end{array}\right]
\end{aligned}
$$

where the subscript $\infty$ indicates the steady state value, and the matrices

$$
\begin{aligned}
& \tilde{A}:=\left[\begin{array}{cc}
A & 0 \\
-C & 0
\end{array}\right], \quad \widetilde{B}:=\left[\begin{array}{c}
B \\
0
\end{array}\right], \quad \tilde{D}:=\left[\begin{array}{l}
D \\
0
\end{array}\right] \\
& \tilde{C}:=\left[\begin{array}{ll}
C & 0
\end{array}\right], \quad \tilde{E}:=\left[\begin{array}{ll}
E & 0
\end{array}\right]
\end{aligned}
$$

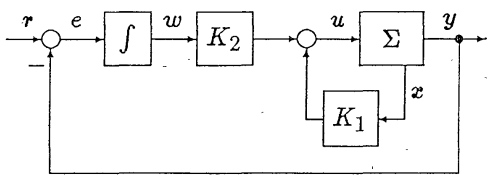

Fig. 1 The structure of the optimal servosystem then we have the following augmented system

$$
\begin{aligned}
& \Sigma_{a}: \delta \dot{\tilde{x}}=(\tilde{A}+\tilde{D} F \tilde{E}) \delta \tilde{x}+\tilde{B} \delta u \\
& \delta y=\tilde{C} \delta \tilde{x}
\end{aligned}
$$

It is well known that if there exists a stabilizing controller for the augmented system, $\Sigma_{a}$ in Eq. (14), we can construct a servosystem for Eq. (10) whose output, $y$, tracks a constant reference input. Furthermore if such a stabilizing controller is LQ optimal, then the servosystem will have low sensitivity to the perturbation of the plant parameters, because the closed loop system applying the LQ optimal controller has this property.

From the Fact 1, it follows that if the augmented system, $\Sigma_{a}$, is quadratically stabilizable, then we can construct the servosystem which is stable, tracks a constant reference input, and has low sensitivity to the perturbation of the plant parameters for each $F \in \mathbf{F}^{p \times q}$.

Applying Lemma 1 and Fact 1 to $\Sigma_{a}$, the following proposition is obtained.

《Proposition 1$\rangle$ Let $\tilde{Q}, \tilde{R}$ be positive definite symmetric matrices. If there exists a constant $\varepsilon>0$ such that the Riccati equation

$$
\begin{aligned}
\tilde{P} \tilde{A} & +\tilde{A}^{T} \tilde{P}-\frac{1}{\varepsilon} \tilde{P} \tilde{B} \tilde{R}^{-1} \tilde{B}^{T} \tilde{P}+\tilde{P} \tilde{D} \tilde{D}^{T} \tilde{P} \\
& +\tilde{E}^{T} \tilde{E}+\varepsilon \widetilde{Q}=0
\end{aligned}
$$

has a positive definite symmetric solution, $\widetilde{P}$

$$
\tilde{P}:=\left[\begin{array}{ll}
P_{11} & P_{12} \\
P_{12}^{T} & P_{22}
\end{array}\right],
$$

then the optimal servosystem with quadratic stability which is optimal for all $F \in \mathbf{F}^{p \times q}$ can be constructed. Further if this condition is satisfied then an optimal control law is obtained as follows

$$
\begin{aligned}
\delta u & =K_{1} \delta x+K_{2} \delta w \\
& =-\frac{1}{\varepsilon} \widetilde{R}^{-1} B^{T} P_{11} \delta x-\frac{1}{\varepsilon} \widetilde{R}^{-1} B^{T} P_{12} \delta w,
\end{aligned}
$$

with the associated quadratic cost function

$$
\begin{aligned}
J_{a}=\int_{0}^{\infty}\{\delta & \left.\tilde{x}^{T} Q_{a P}(F) \delta \tilde{x}+\varepsilon \delta u^{T} \tilde{R} \delta u\right\} d t, \\
Q_{a P}(F):= & \left(\widetilde{P} \tilde{D}-\tilde{E}^{T} F^{T}\right)\left(\widetilde{P} \tilde{D}-\tilde{E}^{T} F^{T}\right)^{T} \\
& +\tilde{E}^{T}\left(I-F^{T} F\right) \tilde{E}+\varepsilon \widetilde{Q}>0 .
\end{aligned}
$$

\section{An Optimal Servosystem with a Set of Tuning Parameters}

As described in Section 3, the servosystem with quadratic stability has a superiority over usual op- 
timal servosystems in robustness. On the other hand, in usual case the weighting matrices of the cost function can be chosen to shape time-response. However, in the case of the servosystem in Section 3 its weighting matrices, $Q_{a P}(F), \varepsilon R$, depend upon $\varepsilon, \tilde{P}$ and $F$, which can not be chosen arbitrary. So in this section we propose a particular servosystem which has quadratic stability and a set of tuning parameters. This servosystem will be obtained by extending the one suggested in Ref. 3) to the uncertain system.

Throughout this section, it is assumed that the nominal system of $\Sigma(F=0)$ is asymptotically stable, that is, $A$ is a stable matrix $A_{s}$, and that the nominal system has no zero at the origin, that is,

$$
\operatorname{det}\left[\begin{array}{cc}
A_{s} & B \\
C & 0
\end{array}\right] \neq 0 \text {. }
$$

Clearly the latter assumption is a necessary condition so that one can construct a servosystem for the nominal system.

\subsection{Condition for Construction of a Par- ticular Servosystem}

First we have the following lemma.

《Lemma 3》 The following conditions are equivalent,

i)

$$
\left\|E\left(s I-A_{s}\right)^{-1}(B L-D)\right\|_{\infty}<1,
$$

where $L:=\left(C A_{s}^{-1} B\right)^{-1} C A_{s}^{-1} D$.

ii) There exist positive definite symmetric matrices $Q_{1}, R_{1}$ such that the Riccati equation $P_{1} A_{s}+A_{s}^{T} P_{1}+E^{T} E+Q_{1}$

$$
+P_{1}(B L-D)\left(I+L^{T} R_{1}^{-1} L\right)(B L-D)^{T} P_{1}=0
$$

has a positive definite symmetric solution $P_{1}$.

iii) There exist positive definite symmetric matrices $P_{1}, W$, and $\widetilde{R}$ such that

$$
\tilde{P}:=\left[\begin{array}{cc}
I & 0 \\
C A_{s}^{-1} & I
\end{array}\right]^{T}\left[\begin{array}{cc}
P_{1} & 0 \\
0 & W
\end{array}\right]\left[\begin{array}{cc}
I & 0 \\
C A_{s}^{-1} & I
\end{array}\right]>0
$$

is a positive definite symmetric solution of the Riccati inequality

$$
\begin{aligned}
& \tilde{P} \tilde{A}_{s}+\tilde{A}_{s}^{T} \tilde{P}-\tilde{P} \tilde{B} \tilde{R}^{-1} \tilde{B}^{T} \tilde{P}+\tilde{P} \tilde{D} \tilde{D}^{T} \tilde{P} \\
& \quad+\tilde{E}^{T} \tilde{E}<0,
\end{aligned}
$$

where $\tilde{B}, \tilde{D}$ and $\tilde{E}$ are defined in Section 3 and

$$
\tilde{A}_{s}:=\left[\begin{array}{cc}
A_{s} & 0 \\
-C & 0
\end{array}\right] \text {. }
$$

The proof of Lemma 3 is shown in Appendix 1.

From this lemma and Proposition 1, we can readily have the following theorem.

《Theorem 1》 Suppose the nominal system of the unsertain system $\Sigma$ is asymptotically stable and satisfies Eq. (20). If $\Sigma$ satisfies Eq. (21), then the servosystem with quadratic stability and a set of tuning parameters, $W>0$, can be constructed. Further if such a condition is satisfied, then the optimal control law with a tuning parameter $W$ is obtained as follows,

$$
\begin{aligned}
\delta u= & -\tilde{R}^{-1} B^{T}\left\{P_{1}+\left(C A_{s}^{-1}\right)^{T} W C A_{s}^{-1}\right\} \delta x \\
& -\tilde{R}^{-1} B^{T}\left(C A_{s}^{-1}\right)^{T} W \delta w,
\end{aligned}
$$

where $P_{1}$ is a positive definite symmetric solution of Eq. (22), and $\tilde{R}$ is defined to satisfy

$$
\tilde{R}^{-1}=R_{1}+L L^{T} \text {. }
$$

And its associated quadratic cost function is

$$
J_{a}=\int_{0}^{\infty}\left\{\delta \tilde{x}^{T} Q_{a P}(F) \delta \tilde{x}+\delta u^{T} \tilde{R} \delta u\right\} d t
$$

where

$$
\begin{aligned}
Q_{a P}(F):= & \left(\tilde{P} \tilde{D}-\tilde{E}^{T} F^{T}\right)\left(\tilde{P} \tilde{D}-\tilde{E}^{T} F^{T}\right)^{T} \\
& +\tilde{E}^{T}\left(I-F^{T} F\right) \tilde{E}+\tilde{Q}>0 \\
\tilde{Q}:= & {\left[\begin{array}{cc}
I & 0 \\
C A_{s}^{-1} & I
\end{array}\right]^{T}\left[\begin{array}{c}
P_{1}\left(B \tilde{R}^{-1}-D L^{T}\right) R_{1}^{-\frac{1}{2}} \\
W C A_{s}^{-1} B R_{1}^{\frac{1}{2}}
\end{array}\right] } \\
& \times\left[\begin{array}{c}
P_{1}\left(B \tilde{R}^{-1}-D L^{T}\right) R_{1}^{-\frac{1}{2}} \\
W C A_{s}^{-1} B R_{1}^{\frac{1}{2}}
\end{array}\right]^{T}\left[\begin{array}{cc}
I & 0 \\
C A_{s}^{-1} & I
\end{array}\right] \\
& +\left[\begin{array}{cc}
Q_{1} & 0 \\
0 & 0
\end{array}\right]>0 .
\end{aligned}
$$

(Proof) From Lemma 3, condition i) is equivalent to ii), iil). So, if condition i) holds, then, for a proper choice of $\tilde{R}$, we have a positive definite symmetric solution $\tilde{P}$ as Eq. (23) to the Riccati inequality (24). Here define $P_{1}$ as a positive definite symmetric solution of Eq. (22), $\tilde{R}$ as Eq. (26), and $\tilde{Q}$ as Eq. (29) then the equality,

$$
\begin{aligned}
& \widetilde{P} \tilde{A}_{s}+\widetilde{A}_{s}^{T} \tilde{P}-\tilde{P} \tilde{B} \tilde{R}^{-1} \widetilde{B}^{T} \tilde{P}+\widetilde{P} \widetilde{D} \tilde{D}^{T} \widetilde{P} \\
& \quad+\tilde{E}^{T} \tilde{E}+\widetilde{Q}=0,
\end{aligned}
$$

holds. This means that when $\tilde{Q}$ is defined as Eq. (29), $\tilde{R}$ to satisfy Eq. (26) and $\varepsilon$ is equal to 1 , the Riccati equation (15) $\left(A=A_{s}\right)$ has a positive definite symmetric solution Eq. (23). Hence from Proposition 1, it can be said that the optimal servosystem with quadratic stability can be constructed and substituting Eq. (23) to Eq. (17), we can get an optimal control law as Eq. (25). Further from Eq. (18) and Eq. (19), it can be seen that its quadratic cost function is obtained as Eqs. (27) (29). 
From Lemma $3, W$ is an arbitrary positive definite symmetric matrix, so we can say that the optimal servosystem has a tuning parameter, $W>0$.

$$
\text { (Q. E. D.) }
$$

The meaning of the tuning parameter, $W$, will be discussed in Section 4.2.

It is easy to show that if the uncertainty, $D F E$, satisfies so-called 'matching condition' (defined below), which is well-known as a sufficient condition for quadratic stabilizability of $\Sigma$, then the augmented system is also quadratically stabilizable; the servosystem with quadratic stability can be constructed. Next, we will show that if the matching condition is satisfied then the conditions in Lemma 3 hold.

【Definition 3】 The uncertainty $D F E$ is said to satisfy the matching condition if there exists a continuous matrix function $N(F)$ such that

$$
D F E=B N(F) \quad \forall F \in \mathbf{F}^{p \times q} .
$$

《Lemma 4$\rangle$ If $D F E$ satisfies the matching condition then the equality

$$
D=B L
$$

holds, where $L$ is a matrix defined in Lemma 3 .

The proof of Lemma 4 is shown in Appendix 2.

From Lemma 4, it is clear that if $\Sigma$ satisfies the matching condition then the condition i) in Lemma 3 holds. Substitute $D=B L$ to Eqs. (22) and (29), then we have the following corollary.

[Corollary 1] Suppose $\Sigma$ satisfies the matching condition. Then the optimal servosystem with a set of parameters, $W>0$, as well as quadratic stability can be constructed. Further define $P_{1}$ as a positive definite symmetric solution of the Lyapunov equation

$$
P_{1} A_{s}+A_{s}^{T} P_{1}+Q_{1}=0,
$$

where $Q_{1}$ is a given positive definite symmetric matrix, $\widetilde{R}$ as a positive definite symmetric matrix which satisfies

$$
\tilde{R}^{-1}-L L^{T}>0,
$$

then the optimal control law is obtained as Eq. (25). And its quadratic cost function is obtained as Eqs. (27), (28) where $\tilde{Q}$ is

$$
\begin{aligned}
\tilde{Q}:= & {\left[\begin{array}{cc}
I & 0 \\
C A_{s}^{-1} & I
\end{array}\right]^{T}\left[\begin{array}{c}
P_{1} \\
W C A_{s}^{-1}
\end{array}\right] B\left(\tilde{R}^{-1}-L L^{T}\right) B^{T} } \\
& \times\left[\begin{array}{c}
P_{1} \\
W C A_{s}^{-1}
\end{array}\right]^{T}\left[\begin{array}{cc}
I & 0 \\
C A_{s}^{-1} & I
\end{array}\right]+\left[\begin{array}{cc}
Q_{1} & 0 \\
0 & 0
\end{array}\right] .
\end{aligned}
$$

From Corollary 1 and the numerical example shown later, we can say that the conditions in Lemma 3 are weaker than the matching condition.

When the nominal system of $\Sigma$ is not stable, before applying Theorem 1 or Corollary 1, we apply pre-feedback, $K_{s}$, in such a way that $A_{s}:=A-B K_{s}$ is stable. If the matching condition holds, then $K_{s}$ can be chosen as an arbitrary stabilizing state feedback law of the nominal system. But if the matching condition does not hold, it must be noted that whether the conditions in Lemma 3 is satisfied or not might depend upon the choice of $K_{s}$.

\subsection{Block Diagram of a Particular Optimal Servosystem}

The optimal servosystem obtained by Theorem 1 is shown in a block diagram, Fig. 2, where the following notation is used

$$
\begin{aligned}
& H_{1}=C A_{s}^{-1}, \\
& H_{2}=-\tilde{R}^{-1} B^{T} P_{1}, \\
& G=-\tilde{R}^{-1}\left(C A_{s}^{-1} B\right)^{T} .
\end{aligned}
$$

Here we have

$$
\begin{aligned}
P_{1} A_{s} & +A_{s}^{T} P_{1}-P_{1} B \tilde{R}^{-1} B^{T} P_{1}+P_{1} D D^{T} P_{1}+E^{T} E \\
= & -P_{1}\left(B \tilde{R}^{-1}-D L^{T}\right) R_{1}^{-1}\left(B \tilde{R}^{-1}-D L^{T}\right)^{T} P_{1} \\
& -Q_{1}<0 .
\end{aligned}
$$

Thus $\mathrm{H}_{2}$ is a quadratically stabilizing control for $\Sigma$. From above, we can say that $W$ is a parameter that tunes the open-loop gain of the system quadrat. ically stabilized by $\mathrm{H}_{2}$.

\section{3 Numerical Example}

In this section we show a numerical example to construct the optimal servosystem with quadratic stability using Theorem 1 .

Consider an uncertain system $\Sigma$ where

$$
\begin{aligned}
& A_{s}=\left[\begin{array}{rr}
-1 & 1 \\
0 & -2
\end{array}\right], \quad B=\left[\begin{array}{l}
1 \\
0
\end{array}\right], \quad C=\left[\begin{array}{ll}
1 & 0
\end{array}\right], \\
& D=\left[\begin{array}{l}
3 \\
1
\end{array}\right], \quad E=\left[\begin{array}{ll}
1 & 1
\end{array}\right] .
\end{aligned}
$$

Note that this system does not satisfy the match-

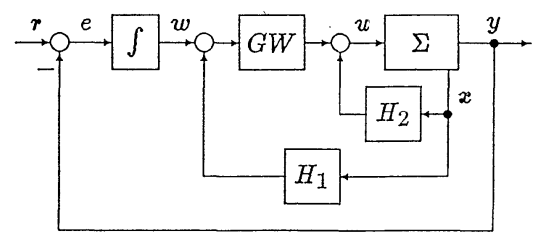

Fig. 2 A particular optimal servosystem with quadratic stability 
ing condition.

We have

$$
\begin{aligned}
& L=\left(C A_{s}^{-1} B\right)^{-1} C A_{s}^{-1} D=\frac{7}{2}, \\
& \left\|E\left(s I-A_{s}\right)^{-1}(B L-D)\right\|_{\infty} \\
& =\left\|-\frac{1}{2(s+1)}\right\|_{\infty}=\frac{1}{2}<1 .
\end{aligned}
$$

Thus the condition i) in Lemma 3 is satisfied. Therefore we have a positive definite symmetric solution of Eq. (22). In fact, for a particular selection of $R_{1}$ and $Q_{1}$

$$
R_{1}=5, \quad Q_{1}=\left[\begin{array}{rr}
0.8992 & -0.2911 \\
-0.2911 & 0.1593
\end{array}\right],
$$

$P_{1}$ is obtained as follows

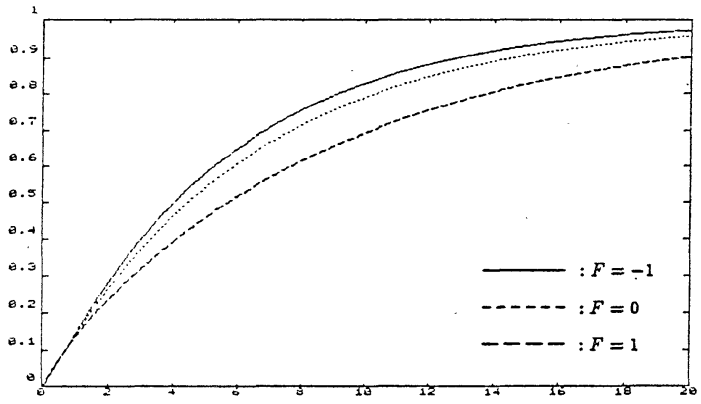

(a) Servosystem $\left(W=\frac{1}{5}\right)$

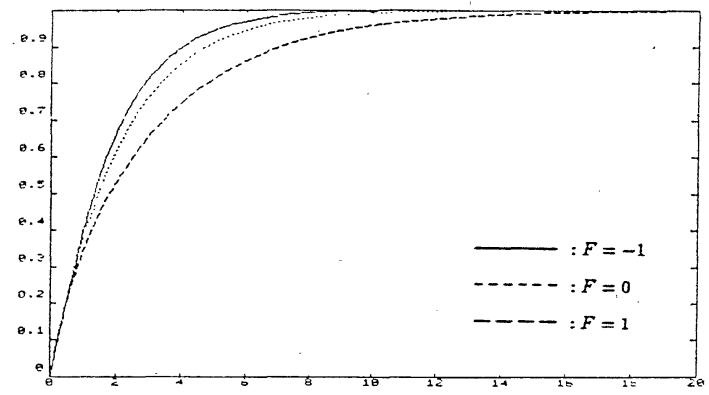

(b) Servosystem ( $W=1)$

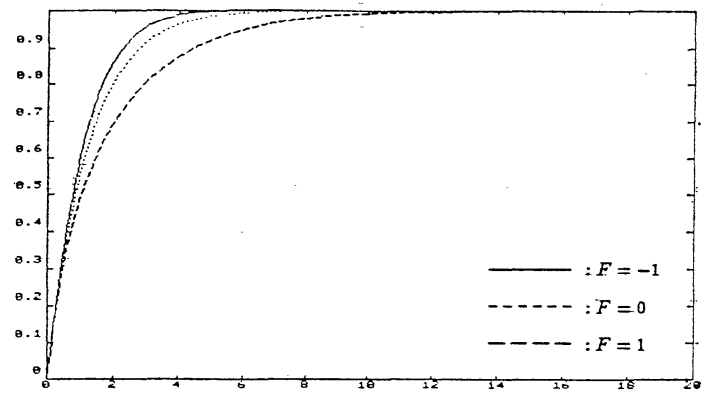

(c) Servosystem $(W=5)$

Fig. 3 Step response of a particular

$$
P_{1}=\left[\begin{array}{ll}
1.0206 & 0.7131 \\
0.7131 & 0.9424
\end{array}\right]
$$

From Eq. (23), we have

$$
\tilde{P}=\left[\begin{array}{ccc}
1.0206+W & 0.7131+\frac{W}{2} & -W \\
0.7131+\frac{W}{2} & 0.9424+\frac{W}{4} & -\frac{W}{2} \\
-W & -\frac{W}{2} & W
\end{array}\right],
$$

$\widetilde{R}^{-1}=17.25$.

$H_{1}, H_{2}$ and $G$ are obtained in the following

$$
\begin{aligned}
& H_{1}=\left[-1-\frac{1}{2}\right], H_{2}=[-17.6049-12.3015] \\
& G=17.25
\end{aligned}
$$

Step responses of the servosystem are shown in Fig. 3.

From above it can be seen that the servosystem has low sensitivity and that, by tuning $W$, we can improve its time-respose.

\section{Conclusion}

In this paper we proposed a servosystem which has quadratic stability (Fig. 1). If, for the augmented system, Petersen's algorithm ${ }^{2}$ (Lemma 1) succeeds, then such a servosystem can be constructed and the control law can be obtained as Eq. (17). Because the servosystem is LQ-optimal for all uncertainties $(F)$ in a given set $\left(\mathbf{F}^{p \times q}\right)$, we can say that it has low sensitivity for all $F \in \mathbf{F}^{p \times q}$. But as mentioned in Section 4, this system is not necessarily fit for designing the time-response. So we proposed a particular servosystem shown in Fig. 2 which has a gain tuning parameter as well as quadratic stability. This system can be constructed if the condition given in Lemma 3 holds. Clearly this condition is only a sufficient condition for quadratic stability of the augmented system but it is weaker than the matching condition.

Fujii, Tsujino and Uematsu' ${ }^{6)}$ also discussed the design of servosystem with quadratic stability. They adopted the inverse LQ design in contrast to the ordinary LQ design in the present work. Our tuning parameters are the gain matrix, $W$, while theirs are pole location and the trade-off parameters in the inverse LQ design method.

As we mentioned in Section 4.1, if the nominal 
system is not stable, the proper selection of the pre-feedback, $K_{s}$, is crucial. So it is desirable to find ways to choose $K_{s}$ so that the system satisfies the condition in Theorem 1.

\section{References}

1) B. D. O. Anderson and J.B. Moore: Linear Optimal Control, Electrical Engineering Series, Prentice-Hall (1971)

2) I. R.Petersen: A Stabilization Algorithm for a Class of Uncertain Linear Systems, Syst. Contr. Letters, 8, 351/357 (1987)

3) N. Suda and M. Ikeda: On Optimal Servosystems, Preprints, IFAC Workshop on System Structure and Control: State-Space and Polynomial Methods, Prague, 157/160 (1989)

4) B.R. Barmish: Necessary and Sufficient Conditions for Quadratic Stabilizability of an Uncertain System, J. Optim. Theory Applic., 46-4, 399/408 (1985)

5) P. P. Khargoneker, I. R. Petersen and K. Zhou: Robust Stabilization of Uncertain Linear Systems: Quadratic Stabilizability and $H_{\infty}$ Control Theory, IEEE Trans. Automatic Control, AC- $-35-3,356 / 361$ (1990)

6) T. Fujii, T. Tsujino and H. Uematsu: An Inverse LQ Based Approach to the Design of Robust Tracking Controllers for Linear Uncertain Systems, Preprints of 19th SICE Symposium on Control Theory, 17/22 (1990) (in Japanese)

\section{《Appendix 1 The Proof of Lemma 3$\rangle$}

In the proof, we use the following lemmata,

《Lemma A. 1》 $\left(\right.$ Petersen $\left.{ }^{2)}\right)$ Let $S$ be a symmetric matrix. Then given any $x \in \mathbf{R}^{n}$, the following equality holds,

$$
\max _{F \in \mathbf{F}^{p \times q}}\left(x^{T} S D F E x\right)=x^{T} S D D^{T} S x x^{T} E^{T} E x .
$$

《Lemma A. 2〉(Petersen $\left.{ }^{2)}\right)$ Let $X, Y$ and $Z$ be given $r \times r$ symmetric matrices such that $X \geq 0$, $Y<0$ and $Z \geq 0$. Further, assume that

$$
\left(\xi^{T} Y \xi\right)^{2}-4\left(\xi^{T} X \xi \xi^{T} Z \xi\right)>0
$$

for all $\xi \in \mathbf{R}^{r}$ with $\xi \neq 0$. Then there exists : a constant $\lambda>0$ such that

$$
\lambda^{2} X+\lambda Y+Z<0 \text {. }
$$

(Proof of Lemma 3)

i ) $\Rightarrow$ ii ) From Lemma 2, if i) holds, then the autonomous system

$$
\dot{x}=\left\{A_{s}+(B L-D) F E\right\} x \quad F \in \mathbf{F}^{p \times q}
$$

is quadratically stable. Then it follows from the definition that there exists a positive definite symmetric matrix $S$ such that

$$
\begin{gathered}
S\left\{A_{s}+(B L-D) F E\right\}+\left\{A_{s}+(B L-D) F E\right\}^{T} S<0, \\
\quad \forall F \in \mathbf{F}^{p \times q} .
\end{gathered}
$$

Therefore

$$
\begin{gathered}
x^{T}\left(S A_{s}+A_{s}^{T} S\right) x<-2 x^{T} S(B L-D) F E x, \\
\forall F \in \mathbf{F}^{p \times q} \text { and }{ }^{\forall} x \in \mathbf{R}^{n}, \quad x \neq 0 .
\end{gathered}
$$

It follows that

$$
\begin{aligned}
& x^{T}\left(S A_{s}+A_{s}^{T} S\right) x \\
& <-2 \max _{F \in \mathrm{F}^{\times \times q}} x^{T} S(B L-D) F E x \leq 0, \\
& \quad \forall x \in \mathbf{R}^{n}, \quad x \neq 0 .
\end{aligned}
$$

Therefore

$$
\begin{aligned}
& \left\{x^{T}\left(S A_{s}+A_{s}^{T} S\right) x\right\}^{2} \\
& >4 \max _{F \in \mathbf{F}^{p \times q}}\left(x^{T} S(B L-D) F E x\right)^{2}, \\
& \quad{ }^{\forall} x \in \mathbf{R}^{n}, \quad x \neq 0 .
\end{aligned}
$$

Using Lemma A.1, we have the following inequality.

$$
\begin{aligned}
& \left\{x^{T}\left(S A_{s}+A_{s}^{T} S\right) x\right\}^{2} \\
& >4 x^{T} S(B L-D)(B L-D)^{T} S x x^{T} E^{T} E x, \\
& \quad{ }^{\forall} x \in \mathbf{R}^{n}, \quad x \neq 0 .
\end{aligned}
$$

Hence from Lemma A. 2, there exists a constant $\lambda>0$ such that

$$
\begin{aligned}
& \lambda^{2} S(B L-D)(B L-D)^{T} S+\lambda\left(S A_{s}+A_{s}^{T} S\right) \\
& \quad+E^{T} E<0 .
\end{aligned}
$$

Define $P_{1}:=\lambda S$, then the above inequality becomes

$$
\begin{aligned}
-\bar{Q}_{1}:= & P_{1} A_{s}+A_{s}^{T} P_{1}+P_{1}(B L-D) \\
& \cdot(B L-D)^{T} P_{1}+E^{T} E<0 .
\end{aligned}
$$

Clearly there exists a positive definite symmetric matrix $R_{1}$ such that

$$
\begin{aligned}
Q_{1} & :=\bar{Q}_{1}-P_{1}(B L-D) L^{T} R_{1}^{-1} L(B L-D)^{T} P_{1} \\
& >0 .
\end{aligned}
$$

From Eqs. (A.11) and (A.12), we have

$$
\begin{aligned}
& P_{1} A_{s}+A_{s}^{T} P_{1}+P_{1}(B L-D)\left(I+L^{T} R_{1}^{-1} L\right) \\
& \cdot(B L-D)^{T} P_{1}+E^{T} E+Q_{1}=0,
\end{aligned}
$$

where $P_{1}>0, R_{1}>0, Q_{1}>0$.

ii) $\Rightarrow$ i ) Suppose there exists a positive definite symmetric solution $P_{1}$ to Eq. (22). Then, we get

$$
\begin{aligned}
x^{T}[ & P_{1}\left\{A_{s}+(B L-D) F E\right\} \\
& \left.+\left\{A_{s}+(B L-D) F E\right\}^{T} P_{1}\right] x \\
= & x^{T}\left[P_{1} A_{s}+A_{s}^{T} P_{1}+P_{1}(B L-D) F E\right. \\
& \left.+\{(B L-D) F E\}^{T} P_{1}\right] x \\
= & x^{T}\left[P_{1} A_{s}+A_{s}^{T} P_{1}+P_{1}(B L-D)(B L-D)^{T} P_{1}\right. \\
& +E^{T} E-\left\{P_{1}(B L-D)-E^{T} F^{T}\right\} \\
& \left.\times\left\{P_{1}(B L-D)-E^{T} F^{T}\right\}^{T}-E^{T}\left(I-F^{T} F\right) E\right] x \\
\leq & x^{T}\left[P_{1} A_{s}+A_{s} P_{1}\right. \\
& \left.+P_{1}(B L-D)(B L-D)^{T} P_{1}+E^{T} E\right] x \\
= & x^{T}\left[-Q_{1}-P_{1}(B L-D) L^{T} R_{1}^{-1} L(B L-D)^{T} P_{1}\right] x
\end{aligned}
$$




$$
\begin{aligned}
& \leqq-x^{T} Q_{1} x \\
& \leqq-\alpha\|x\|^{2}, \quad \forall F \in \mathbf{F}^{p \times q}, \quad{ }^{\forall} x \neq 0 \in \mathbf{R}^{n}
\end{aligned}
$$

where $\alpha>0$ indicates minimum singular value of $Q_{1}$.

Above inequality implies that the autonomous system (A.4) is quadratically stable. Then from Lemma 2, we have

$$
\left\|E\left(s L-A_{s}\right)^{-1}(B L-D)\right\|_{\infty}<1 .
$$

iii) $\Rightarrow$ ii ) From Eqs. (23), (24), we get

$$
\begin{aligned}
& \tilde{P} \tilde{A}_{s}+\tilde{A}_{s}^{T} \tilde{P}-\tilde{P} \tilde{B} \tilde{R}^{-1} \tilde{B}^{T} \tilde{P}+\tilde{P} \tilde{D} \tilde{D}^{T} \tilde{P}+\tilde{E}^{T} \tilde{E} \\
& =-\left[\begin{array}{cc}
I & 0 \\
C A_{s}^{-1} & I
\end{array}\right]^{T} \tilde{Q}\left[\begin{array}{cc}
I & 0 \\
C A_{s}^{-1} & I
\end{array}\right]<0, \quad(\text { A. 16) }
\end{aligned}
$$

where

$$
\bar{Q}:=\left[\begin{array}{ll}
Q_{11} & Q_{12} \\
Q_{12}^{T} & Q_{22}
\end{array}\right]
$$

is the matrix determined by

$$
\begin{aligned}
Q_{11}= & -\left(P_{1} A_{s}+A_{s}^{T} P_{1}-P_{1} B \widetilde{R}^{-1} B^{T} P_{1}\right. \\
& \left.+P_{1} D D^{T} P_{1}+E^{T} E\right), \\
Q_{12}= & P_{1}\left(B \widetilde{R}^{-1}-D L^{T}\right)\left(C A_{s}^{-1} B\right)^{T} W, \\
Q_{22}= & W C A_{s}^{-1} B\left(\widetilde{R}^{-1}-L L^{T}\right)\left(C A_{s}^{-1} B\right)^{T} W .
\end{aligned}
$$

Since $\bar{Q}>0$, we have

$$
\begin{aligned}
& Q_{22}>0, \\
& Q_{11}-Q_{12} Q_{22}^{-1} Q_{12}^{T}>0 .
\end{aligned}
$$

From Eqs. (20) and (A. 19), we get easily that $C A_{s}^{-1} B$ is non-singular and that $\tilde{R}^{-1}>L L^{T}$. It follows from this and Eq. (A. 20) that

$$
\begin{aligned}
& P_{1} A_{s}+A_{s}^{T} P_{1}-P_{1} B \widetilde{R}^{-1} B^{T} P_{1}+P_{1} D D^{T} P_{1}+E^{T} E \\
& \quad+P_{1}\left(B \widetilde{R}^{-1}-D L^{T}\right)\left(\tilde{R}^{-1}-L L^{T}\right) \\
& \quad \times\left(B \widetilde{R}^{-1}-D L^{T}\right)^{T} P_{1}<0 .
\end{aligned}
$$

Define

$$
R_{1}:=\widetilde{R}^{-1}-L L^{T}>0 .
$$

Then we get

$$
\begin{aligned}
P_{1} A_{s} & +A_{s}^{T} P_{1}-P_{1} B \tilde{R}^{-1} B^{T} P_{1}+P_{1} D D^{T} P_{1}+E^{T} E \\
& +P_{1}\left(B \tilde{R}^{-1}-D L^{T}\right)\left(\tilde{R}^{-1}-L L^{T}\right) \\
& \times\left(B \widetilde{R}^{-1}-D L^{T}\right)^{T} P_{1} \\
= & P_{1} A_{s}+A_{s}^{T} P_{1}+P_{1}(B L-D)\left(I+L^{T} R_{1}^{-1} L\right) \\
& \cdot(B L-D)^{T} P_{1}+E^{T} E<0 .
\end{aligned}
$$

In establishing above inequality, we use

$$
\begin{aligned}
- & B \widetilde{R}^{-1} B^{T}+D D^{T}+\left(B \tilde{R}^{-1}-D L^{T}\right) \\
& \cdot\left(\tilde{R}^{-1}-L L^{T}\right)^{-1} \times\left(B \widetilde{R}^{-1}-D L^{T}\right)^{T} \\
= & B L L^{T} B^{T}-D L^{T} B^{T}-B L D^{T}+D D^{T} \\
& +B L L^{T} R_{1}^{-1} L L^{T} B^{T}-B L L^{T} R_{1}^{-1} L D^{T} \\
& -D L^{T} R_{1}^{-1} L L^{T} B^{T}+D L^{T} R_{1}^{-1} L D^{T} \\
= & (B L-D)(B L-D)^{T}
\end{aligned}
$$

$$
\begin{aligned}
& +(B L-D) L^{T} R_{1}^{-1} L(B L-D)^{T} \\
= & (B L-D)\left(I+L^{T} R_{1}^{-1} L\right)(B L-D)^{T} . \quad \text { (A. 24) }
\end{aligned}
$$

It follows that there exists a positive definite symmetric matrix $Q_{1}$ such that the Riccati equation (22) has a positive definite symmetric solution $P_{1}$.

ii $) \Rightarrow \dddot{i i l}$ ) Define $\tilde{R}$ to satisfy Eq. (A.22) and $P_{1}$ as a positive definite symmetric solution of Eq. (22). Then tracing the proof of iii) $\Rightarrow$ ii) conversely, we can easily show that for an arbitrary positive definite symmetric matrix $W, \tilde{P}$ is a positive definite symmetric solution of the matrix inequality (24).

(Q. E. D.)

\section{《Appendix 2 The proof of Lemma 4$\rangle$}

If $\operatorname{rank} B=n$ then the lemma is clearly shown. So we consider the case $\operatorname{rank} B<n$.

Suppose there exists an $n$-dimensional vector $x$ such that

$$
\begin{aligned}
& x \in \operatorname{ker} B^{T}, \\
& x^{T} D \neq 0 .
\end{aligned}
$$

Define

$$
x^{T} D=\left[d_{1}, \cdots, d_{p}\right], \quad d_{r} \neq 0,
$$

where $r$ is an integer in $[1, p]$. On the other hand, without loss of generality, we suppose that the $s$-th row and $t$-th column element of $E, e_{s t}$, is not zero. Now, choose $F \in \mathbf{F}^{p \times q}$ as

$$
\begin{aligned}
& F=\left[f_{i j}\right], \\
& \left\{\begin{array}{l}
f_{r s}=1, \\
f_{i j}=0, \quad i \neq r \text { or } j \neq s .
\end{array}\right.
\end{aligned}
$$

Then we get $x^{T} D F E=\left[*, \cdots, *, d_{r} e_{s t}, *, \cdots, *\right] \neq 0$.

This contradicts the fact

$$
x^{T} D F E=x^{T} B N(F)=0 .
$$

Hence $x^{T} D=0$ for all $x \in \operatorname{ker} B^{T}$. Therefore $\operatorname{ker} B^{T} \subseteq \operatorname{ker} D^{T}$.

So, we have

$$
\operatorname{Im} D \subseteq \operatorname{Im} B .
$$

From this, we can say that there exists a matrix $\bar{L} \in \mathbf{R}^{m \times p}$ such that

$$
D=B \bar{L} \text {. }
$$

From Eq. (A.32), we have

$$
L=\left(C A_{s}^{-1} B\right)^{-1} C A_{s}^{-1} D=\bar{L} \text {. }
$$

Therefore it has shown that if the matching condition holds then $D=B L$.

(Q. E. D.) 